\title{
The ADAMs family of proteases: new biomarkers and therapeutic targets for cancer?
}

Michael J Duffy ${ }^{1,2^{*}}$, Maeve Mullooly ${ }^{1,2}$, Norma O'Donovan ${ }^{3}$, Sumainizah Sukor ${ }^{2,4}$, John Crown ${ }^{4}$, Aisling Pierce ${ }^{1,2}$ and Patricia M McGowan ${ }^{1,2}$

* Correspondence: michael.j. duffy@ucd.ie

${ }^{1}$ Department of Pathology and Laboratory Medicine, St. Vincent's University Hospital, Dublin 4 Ireland

Full list of author information is available at the end of the article

\begin{abstract}
The ADAMs are transmembrane proteins implicated in proteolysis and cell adhesion. Forty gene members of the family have been identified, of which 21 are believed to be functional in humans. As proteases, their main substrates are the ectodomains of other transmembrane proteins. These substrates include precursor forms of growth factors, cytokines, growth factor receptors, cytokine receptors and several different types of adhesion molecules. Although altered expression of specific ADAMs has been implicated in different diseases, their best-documented role is in cancer formation and progression. ADAMs shown to play a role in cancer include ADAM9, ADAM10, ADAM12, ADAM15 and ADAM17. Two of the ADAMs, i.e., ADAM10 and 17 appear to promote cancer progression by releasing HER/EGFR ligands. The released ligands activate HER/EGFR signalling that culminates in increased cell proliferation, migration and survival. Consistent with a causative role in cancer, several ADAMs are emerging as potential cancer biomarkers for aiding cancer diagnosis and predicting patient outcome. Furthermore, a number of selective ADAM inhibitors, especially against ADAM10 and ADAM17, have been shown to have anti-cancer effects. At least one of these inhibitors is now undergoing clinical trials in patients with breast cancer.
\end{abstract}

\section{Review}

The ADAMs are a family of multidomain proteins shown to be involved in both proteolysis and cell adhesion [for review, see refs [1-3]]. Although primarily located on the cell membrane, soluble forms have been described for some ADAMs. The best established role for ADAMs is the activation of the proforms of certain growth factors and cytokines as well as the shedding of the extracellular domains of growth factor receptors and adhesion proteins. ADAMs thus play a role in remodelling or processing of cell membrane proteins. Several of the substrates processed by ADAMs, especially by ADAM10 and ADAM17, have been implicated in the pathogenesis or progression of cancer [for reviews, see refs $[4,5]$ ], though some proteolytically inactive ADAMs may also play important roles in carcinogenesis (summarised in Table 1). The aim of this article is to review the role of ADAMs in malignancy, focusing especially on their potential use as cancer biomarkers and therapeutic targets. Firstly however, we briefly review the protein structure and biological activities of ADAMs.

(C) 2011 Duffy et al; licensee BioMed Central Ltd. This is an Open Access article distributed under the terms of the Creative Commons Attribution License (http://creativecommons.org/licenses/by/2.0), which permits unrestricted use, distribution, and reproduction in any medium, provided the original work is properly cited. 
Table 1 Potential functions of human ADAMs*

\begin{tabular}{ll}
\hline ADAM & Function/potential function \\
\hline $\begin{array}{ll}\text { Proteolytically } \\
\text { inactive }\end{array}$ \\
\hline ADAM2 & Sperm-egg fusion \\
\hline ADAM7 & Sperm maturation \\
\hline ADAM11 & Integrin ligand, neural adhesion, tumour suppressor \\
\hline ADAM18 & Oocyte recognition \\
\hline ADAM22 & Adhesion \\
\hline ADAM23 & Tumour suppressor \\
\hline ADAM29 & Unknown \\
\hline Proteolytically & $\begin{array}{l}\text { Shedding of adhesion molecules, leukocyte receptors, neutrophil infiltration, osteoclast } \\
\text { active* }\end{array}$ \\
\hline ADAM8 & $\alpha$-secretase activity, cellular adhesion \\
\hline ADAM9 & $\begin{array}{l}\alpha \text {-secretase activity, shedding of TNF } \alpha, \text { EGF, betacellulin, HER2, Notch, and collagen IV, } \\
\text { cellular adhesion }\end{array}$ \\
\hline ADAM10 & Cellular adhesion, shedding of HB-EGF \\
\hline ADAM12 & Cellular adhesion \\
\hline ADAM15 & $\begin{array}{l}\text { Release of several growth factor ligands, e.g., TNF-alpha and specific EGFR/HER ligands, } \\
\text { cellular adhesion }\end{array}$ \\
\hline ADAM17 & Unknown \\
\hline ADAM19 & Shedding of IGFBP3 \\
\hline ADAM28 & Involved in pathogenesis of gastric cancer via IL-18 secretion \\
\hline ADAM33 & LPL; lipoprotein lipase, CLL; chronic lymphocytic leukemia, TNF $\alpha$; tumour necrosis factor-alpha, EGF; epidermal growth \\
factor, HB-EGF; heparin & -binding-EGF, IGFBP3; insulin-like growth factor-binding protein 3, IL-18; interleukin-18 \\
\hline
\end{tabular}

\section{Structure of ADAM Proteins}

The generalised structure of an ADAM protein contains 8 distinct domains or regions. In the typical ADAM protein, these domains are a signal domain, a prodomain, a metalloproteinase domain, a disintegrin or integrin-binding domain, a cysteine rich region, an EGF (epidermal growth factor)-like domain, a transmembrane sequence and an intracellular $C$-terminal end [1]. Like most proteases, the ADAMs are initially synthesised as enzymatically-inactive precursor proteins. As with MMPs, this inactive state in most of the ADAMs is due to the interaction of a cysteine residue in the prodomain with the zinc ion at the catalytic site. For protease activation, this prodomain is removed by a furin-like convertase or by autocatalysis, depending on the specific ADAM [1,2]. This cysteine switch mechanism however, does not appear to play a role in maintaining the zymogen state of ADAM17 [6].

Next to the prodomain is the MMP-like domain. Although all ADAMs possess this sequence, only about $50 \%$ exhibit protease activity. Thus, of the 21 human ADAMs identified, only 13 are proteolytically active. ADAMs shown to exhibit protease activity include ADAM9, 10, 12, 15, 17, 19, 28 and 33. Currently, protease activity is the bestdefined function of ADAMs, with most of the putative substrates currently identified being transmembrane proteins.

Downstream of the MMP domain is the disintegrin domain. This sequence, which is found in all ADAMs binds to integrins, a group of adhesion proteins involved in cell 
adhesion, migration and cell signalling [7]. It should be stated that most of the work relating to the binding of disintegrins to integrins has been carried out in vitro using recombinant disintegrin domains [8]. The biological relevance of these findings are thus unclear.

The function(s) of the remaining domains, i.e., the cysteine-rich region, EGF-like sequence and cytoplasmic remain to be determined. In some of the ADAMs however, the cysteine region has been implicated in regulating protease activity and controlling substrate specificity [9]. The C-terminal domain of ADAM17 has been shown to undergo phosphorylation at different sites including $\operatorname{Thr}^{735}$ and $\operatorname{Ser}^{819}$ [10-14]. Thus, phosphorylation at $\mathrm{Thr}^{735}$ was found to be necessary for ADAM17-catalysed shedding of TGF-alpha [12]. With ADAM15, phosphorylation of the cytoplasmic domain resulted in interaction with several potential signalling proteins, including the Src kinases, Hck and Lek [14]. It was unclear whether or not this interaction led to altered intracellular cell signalling.

\section{Role of ADAMs in Cancer Formation and Progression}

\section{Evidence that ADAMs play a causal role in cancer}

Studies from cell lines grown in culture, animal models and human malignancies suggest that a number of ADAMs are involved in cancer formation and/or progression [4,5]. Specific ADAMs implicated in these processes include ADAM9, ADAM10, ADAM12, ADAM15 and ADAM17. Of these different ADAMs, the strongest evidence for a role in malignancy exists for ADAM17, which is also known as TACE (tumour necrosis factor alpha converting enzyme). Briefly, the evidence implicating ADAMs in malignancy is as follows $[4,5]$ :

- Inhibition of ADAM17 activity or downregulation of its expression decreased growth of breast cancer cells in vitro and reversed their morphological appearance to that approximating normal cells [15].

- Several studies have shown that increased expression of certain ADAMs enhanced in vitro invasion, proliferation and promoted tumour formation in vivo [16-21], while decreased expression reduced these processes.

- Deficiency of specific ADAMs such as ADAM9, 15 and 17 resulted in decreased growth of heterotopically injected tumour cells in mice models [22,23].

- Correlations exist between between levels of specific ADAMs and parameters of tumour progresion (eg., tumour size, grade, metastasis to local lymph nodes and patient outcome) in human cancers [26-31] and

- Selective inhibitors against certain ADAMs reduce or block tumour cell growth in model system [32-34].

\section{Mechanisms by which ADAMs play a role in cancer}

Activation of positively-stimulating pathways

ADAMs could potentially promote cancer formation and progression using several different mechanisms. One of the most likely of these involves the release or activation of positively-stimulating growth factors (Figure 1). Many of these growth factors are initially synthesised as inactive transmembrane precursor proteins that require conversion 


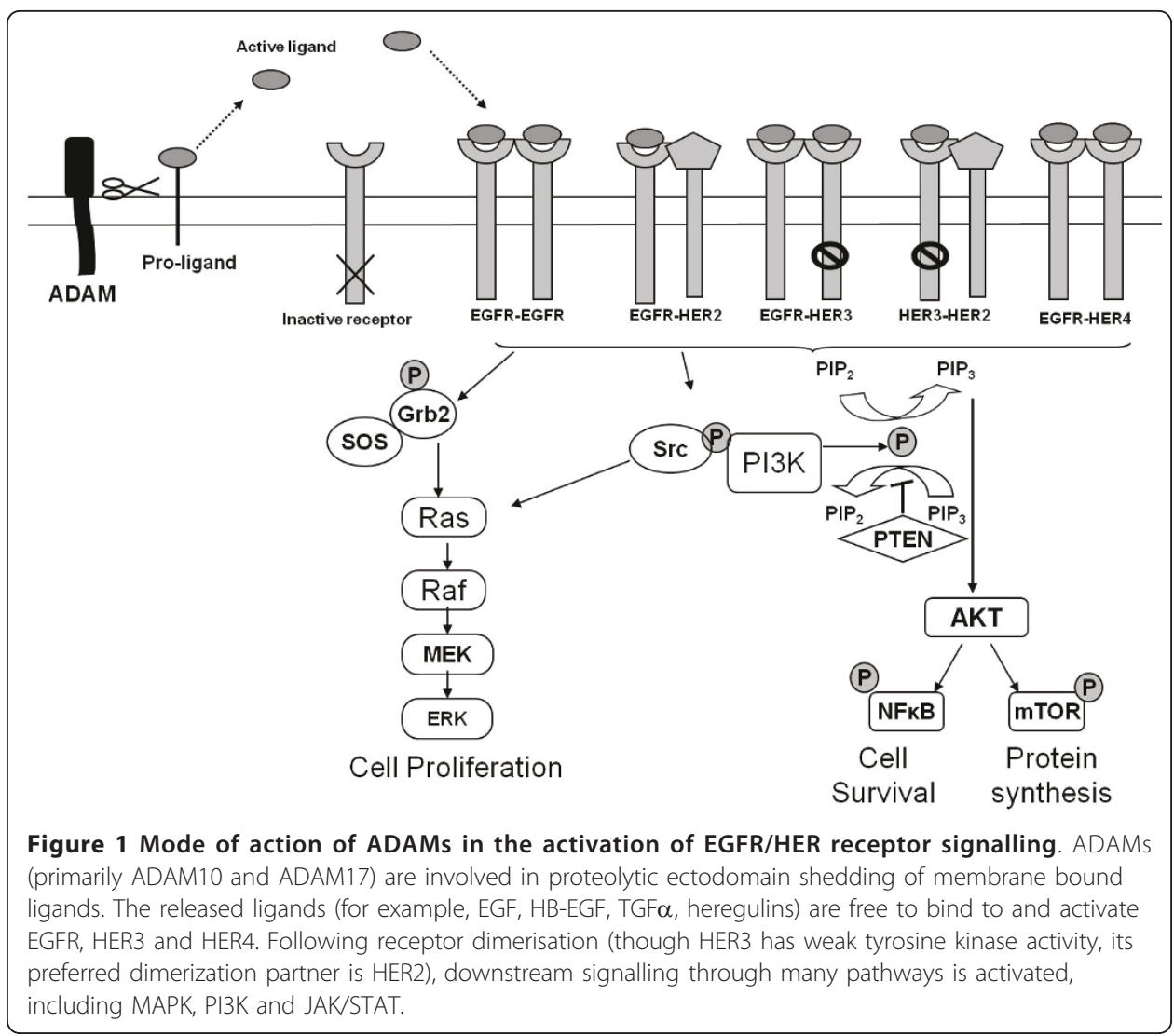

to an active state in order to exert maximun activity. Amongst the best-studied growth-stimulating factors that are activated by ADAMs are the EGFR/HER family of ligands. Conversion of these ligands to their active state is primarily mediated by either ADAM10 or ADAM17. Thus, ADAM17 appears to be the physiological sheddase for TGF-alpha, amphiregulin, HB-EGF, and epiregulin. ADAM10, on the other hand, appears to be the major sheddase for the release of EGF and betacellulin [35,36]. In certain situations however, other ADAMs including ADAM8, 9, 12, 17 and 19 can activate one or more of these ligands [37].

The shed form of these ligands binds to one or more of the EGFR/HER family of receptors. Four members of this family exist, i.e., HER1 (c-erbB1), HER2 (c-erbB2), HER3 (c-erbB3) and HER4 (c-erbB4). These 4 receptors have a similar general structure that includes an extracellular domain, a transmembrane domain and an intracellular domain [38-40]. All of these receptors, apart from HER2, can be directly activated following ligand binding. Following homo- or heterodimerisation, downstream signalling from these receptors activates several different pathways including the mitogenactivated protein kinase (MAPK) pathway, the phosphatidylinositol 3-kinase (PI3K) pathway and janus kinase/signal transducer and activator of transcriptional (JAK/ STAT) pathway. This signalling results in some of the classical hall markers of malignancy such as enhanced cell proliferation, increased cell motility and increased cell survival [38-40].

Substantial evidence implicating ADAM-mediated growth factor ligand release and EGFR signalling in cancer cell proliferation or migration is now available. Singh et al 
[41] showed that UV irradiation of skin cancer cells activated ADAM9 and 17 which was followed by amphiregulin shedding, EGFR transactivation and increased cell proliferation. In another study, Zheng et al [21] reported that ADAM17, via ligand release and activation of the EGFR-PI3K-AKT pathway, enhanced in vitro breast cancer cell proliferation and invasion. In a further study, Mendelson et al [42] showed that treating mouse embryonic fibroblasts with platelet derived growth factor receptor beta (PDGFR $\beta$ ) led to activation of ADAM17, release of EGFR ligands and EGFR/ERK signalling. This cascade of events ultimately resulted in enhanced migration.

While shedding of the extracellular domain of the HER ligands results in receptor binding, at least for heparin binding-epidermal growth factor [HB-EGF], it can also lead to translocation of its $\mathrm{C}$-terminal fragment from the cell membrane into the nucleus and regulation of cell proliferation. This translocation of the C-terminal domain of HB-EGF has been shown both in keratinocytes [43] and gastric cancer cells [44] and is thus another possible mechanism by which ADAM-catalysed shedding of growth factors can alter cell proliferation.

\section{Inactivation of growth-inhibitory pathways}

Inactivation of growth inhibitory signalling systems would be expected to produce the same end result as activation of positively-activating growth factors. One of the best examples of a negatively-acting growth factor is TGF $\beta$ which signals via TGF $\beta$ R1 and TGF $\beta 2$ [45]. In normal and early malignant cells, TGF $\beta$ inhibits proliferation. In contrast in progressive malignancy, TGF $\beta$ promotes proliferation [45]. Recently, ADAM17 was reported to mediate shedding of the type 1 TGF $\beta$ receptor [46]. As a result, TGF $\beta$ signalling was decreased which in turn led to decreased growth inhibition. According to Liu et al [46], this ADAM17 mediated reduction in growth inhibition complements the growth stimulation, resulting from increased release of the EGFR/HER ligands, see above.

\section{Shedding of adhesion proteins}

ADAM-mediated shedding of adhesion proteins may also result in increased cell proliferation. Maretzky et al [47] showed that ADAM10 caused shedding of the extracellular domain of cadherin $\mathrm{E}$, which resulted in the translocation of beta-catenin to the nucleus and enhanced proliferation. In other work, Najy et al [48] found that an ADAM15-mediated shed form of cadherin E bound to and activated HER2 in breast cancer cells [48]. The shed form of cadherin E formed a complex with HER2 and HER3, that gave rise to enhanced ERK signalling, which in turn, led to increased proliferation and migration.

It was mentioned above that shedding of cadherin $\mathrm{E}$ resulted in increased cell proliferation. As well as enhancing cell proliferation, this shedding might also be expected to weaken cell:cell interaction and thus allow dissociation of potential invasive and metastatic cells in the primary cancer. Such dissociation could potentially place a malignant cell or group of cells on their pathway to metastais. Shedding of other adhesion proteins such as L-selectin, ICAM-1 or VCAM, on the other hand, might be expected to modulate binding of tumour cells to the vasculature wall and thus play a role in the intravasation [i.e., exiting of tumour cells from the vasculature into a distant organ].

\section{Potential involvement in mediating angiogenesis}

Finally, ADAMs may promote cancer growth and metastasis by mediating angiogenesis or pathological neovascularisation. Angiogenesis is defined as the protrusion and outgrowth of capillary buds and sprouts from pre-existing blood vessels [49]. This process 
is essential for tumours to grow beyond approximately $2 \mathrm{~mm}$ in diameter. Early evidence implicating ADAMs in angiogenesis was the finding of pulmonary hypovascularisation in mice expressing catalytically inactive ADAM17 [50]. More recently, Gooz et al [51] showed that knockdown of ADAM17 expression using siRNA decreased endothelial cell proliferation and invasion in vitro. Furthermore, in a mouse model, Weskamp et al [25] reported that deletion of ADAM17 resulted in pathological neovascularisation and reduced growth of injected tumour cells. In this animal model, neither developmental nor vascular homeostasis was affected by the loss of ADAM17. Other ADAMs implicated in pathological neovascularisation include ADAM9 [22] and ADAM15 [23,24].

\section{ADAMs as Biomarkers in Cancer}

Biomarkers are potentially useful in cancer detection (screening and aiding diganosis), asssessing prognosis, upfront predicting likely response or resistance to therapy and monitoring ongoing therapy [for review, see ref. 52]. In recent years, several preliminary reports suggested that a number of different ADAMs may act as cancer biomarkers. This evidence is briefly reviewed below.

\section{ADAMs as diagnostic aids in cancer}

For aiding cancer diagnosis, a biomarker should be specifically altered in the majority of patients with a specific malignancy or premalignant condition. Furthermore, it should be measurable in a readily available fluid such as serum or urine. In recent years, a number of ADAMs have been detected in these fluids from patients with cancer. One of the first ADAMs shown to have diagnostic potential was ADAM12 in breast cancer. Using Western blotting, Roy et al [53], reported that urinary levels of ADAM12 were significantly increased in patients with breast cancer vis-à-vis a healthy control group. Furthermore, the proportion of patients with high levels of this ADAM was significantly greater in the breast cancer patients than in the healthy controls. Levels were disease stage-related, progressively increasing from patients with in situ disease, to those with locally invasive disease to those with metastatic disease. Using logistic regression analysis, the authors calculated that the predictive probability of the presence of breast cancer was $\geq 80 \%$, when levels of ADAM12 exceeded 40 arbitrary units [53].

In a follow-up study to above, Pories et al [54] found that urinary ADAM12 levels were also increased in women with putative premalignant lesions of invasive breast cancer such as atypical hyperplasia and lobular carcinoma in situ, compared to levels in healthy controls. This finding, if confirmed, suggests that measurement of ADAM12 in urine could identify women at increase risk of developing breast cancer. Clearly, these preliminary but promising findings, require confirmation in larger studies. It should be stated that none of the available serum markers for breast cancer are increased in patients with early disease and are thus of little value in identifying women at increased risk of developing this malignancy [55].

As well as breast cancer, ADAM12 has also been found to be elevated in urine from patients with bladder cancer, compared with healthy control subjects [56]. Indeed, measurement of ADAM12 appeared to be a more sensitive diganostic marker for bladder cancer that standard cytology. Although levels were increased in patients with early stage disease, including those with superficial non-invasive disease and superficial 
invasive disease, concentrations tended to be higher in those with the largest invasive tumours. In the small number of cases studied, urinary ADAM12 levels decreased following surgical removal of the bladder cancer but increased again with recurrent disease [56]. This latter finding suggests that measurement of urinary ADAM12 may be suitable for monitoring patients with bladder cancer.

One of the first ADAMs shown to be elevated in serum from patients with cancer was ADAM28 [57]. Using ELISA, Kuroda et al [57] found that serum levels of this ADAM in patients with non-small cell lung cancer were approximately 5-fold greater than levels in a healthy control group. As with urinary levels of ADAM12 in breast cancer, serum levels of ADAM28 increased progressively with increasing disease stage. Interestingly, the diagnostic acuracy of ADAM28 appeared to be higher than that of one of the establised marker for non-small cell lung cancer, i.e., carcinoembryonic antigen (CEA).

\section{ADAMs as prognostic markers}

Prognostic markers are important in the management of patients with cancer as they help avoid the overtreatment of indolent disease and undertreatment of aggressive cases. Ideally, a new biological prognostic marker should provide additional or independent information to that available from the conventional factors such as tumour size, tumour grade and metastasis to local lymph nodes. New prognostic markers are most urgently needed for cancers such as breast and prostate cancer. In breast cancer, prognostic markers may help identify those patients whose prognosis is so good that they are unlikely to benefit from receiving adjuvant chemotherapy. The corollory is that the same marker(s) can help identify patients with aggressive disease that may derive benefit from receiving such therapy. As certain ADAMs have been implicated in tumour development and progression, it is not surprising that they have been investigated for potential prognostic impact in patients with cancer.

One of the best validated ADAMs for predicting patient outcome is ADAM17 in breast cancer. Using ELISA, McGowan et al showed that patients with breast cancers expressing high levels of ADAM17 protein had significantly shorter overall survival compared to those with low expression of the protein [31]. Importantly, the prognostic impact of ADAM17 was independent of tumour size, grade and lymph node status. Although the ELISA used in this study detected both the precursor and active forms of ADAM17, a previous study found that the active form was more associated with breast cancer progression than the precursor form [16]. As well as ADAM17 protein, high expression of ADAM17 mRNA was also found to predict adverse outcome in patients with breast cancer [15].

Another ADAM associated with outcome in patients with breast cancer is ADAM15. Four isoforms or variants of this ADAM have been described, ADAM15-A, ADAM15B, ADAM15-C and ADAM15-D. These different forms of ADAM15 have been shown to have different effects in vitro [29]. Thus, ADAM15-A was found to increase cell invasion, migration and adhesion, while ADAM15-B was shown to decrease adhesion. Although these 2 variants had different effects on adhesion in vitro, high expression of both predicted shortened relapse-free survival in lymph node-negative breast cancer patients. ADAM15-C, on the other hand, correlated with improved relapse-free survival in lymph node-positive but not in lymph node-negative patients. 
One of the cancers for which new prognostic markers are most urgently required is prostate cancer. Although prostate cancer is the most common malignancy affecting males, most of these tumours are indolent and never progress to a symptomatic stage. However, a minority are aggressive and rapidly progress causing morbidity and mortality. A major everyday problem in the management of men with newly diagnosed prostate cancer is therefore differentiating men with indolent disease from those with life-threatening disease.

Using immunohistochemistry, Fritzche et al [28], showed that increased expression of ADAM9 in prostate cancer was significantly associated with shortened relapse-free survival as measured by increasing serum PSA levels. As with ADAM17 in breast cancer, the prognostic impact of ADAM9 in prostate cancer was independent of the conventionally used factors for this malignancy such as tumour size, Gleason grade and preoperative PSA level. This independent prognostic impact of ADAM9 was found in both the total population of patients investigated as well as in those treated with anti-androgens [28]. Other malignancies for which ADAM9 has also been shown to have prognostic value include renal [27] and pancreatic cancers [58].

\section{ADAMs as therapy predictive markers}

Predictive markers are factors that are associated with upfront response or resistance to a particular therapy [59]. Predictive markers are important in the management of cancer patients as tumours of the same histological type or tissue of origin vary widely in their response to most available systemic therapies. Ideally, therefore, markers should be available that predicts likely response. Markers that predict resistance however, may also be helpful. In this latter situation, if available, patients could receive an alternative therapy that may be more beneficial. If an effective alternative therapy is unavailable, these patients could volunteer to participate in clinical trials evaluating new therapies or they could make an informed decision to avoid the needless costs and toxicity of likely ineffective therapy [59].

In one of the few studies carried out to date on ADAMs as therapy predictive biomarkers, Siewerts et al [60] reported that high levels of mRNA for ADAM9 and 11 but not for ADAM10 or ADAM12 were associated with increased benefit from tamoxifen in patients with recurrent breast cancer [60]. This finding was especially true for patients whose primary tumour contained large amounts of stroma. ADAM9 but not ADAM11 provided independent predictive information over estrogen receptors, progesterone receptors, menopausal status and dominant site of relapse.

\section{Summary and Conclusion on ADAMs as Cancer Biomarkers}

Although the above studies indicating a cancer biomarker potential for different ADAMs are promising, they all require confirmation in larger and prospective studies. For evaluating a potential cancer diagnostic role for the ADAMs, it is important that control samples are taken from subjects with a relevant benign disease rather from a healthy population. For studies evaluating a prognostic impact, homogenously-managed groups of patients should be investigated. Such a study is best carried out prospectively although a sufficiently high-powered retrospective analysis lacking bias should also provide reliable results. Predictive biomarkers are most conveniently evaluated in either the neoadjuvant or advanced disease settings, i.e., where measurable disease is present. In the adjuvant setting, predictive markers should be investigated as part of a 
randomised trial in which the marker is used to determine response in the treatment arm while a potential prognostic value can be evaluated in the control arm without systemic treatment.

ADAMs as Therapeutic Targets for the Treatment of Cancer

Since considerable evidence from model systems suggest that specific ADAMs are causally involved in cancer formation and progression, it might be expected that inhibition of these proteases could be used to treat cancer. At least 4 potential approaches exist to block ADAM protease activity. These include use of low molecular weight synthetic inhibitors [see below], purified or synthetic forms of ADAM prodomains [61,62], modified TIMPs [63,64] and monoclonal antibodies [65]. Of these potential approaches, only the use of low molecular weight synthetic inhibitors has been subjected to detailed investigation.

Most of the low molecular weight ADAM inhibitors use hydroxamate as the zinc binding group and were designed to bind to the MMP-like catalytic site [66-69]. Although the majority of those described, inhibited a number of MMPs as well as some ADAMs, a small number were relatively selective for specific ADAMs, especially ADAM10 and/or ADAM17 [32-34,70-75] (Table 2). Of the compounds listed in Table 2, the most widely investigated for anticancer activity are INCB3619 and INCB7839 [Incyte Corporation, Wilmington, DE] [32-34,70-72].

INCB3619 is an orally-active low molecular weight molecule that selectively inhibits ADAM10 and ADAM17 with low $\mathrm{IC}_{50}$ values [14 and 22 nmoles/L, respectively] [33]. It has been shown to inhibit tumour cell growth in several different preclinical models. Thus, in an early study, with non small cell lung cancer [NSCLC] cells in culture, INCB3619 was found to block release of the HER3 ligand, heregulin, rendering these cells sensitive to the EGFR inhibitor, gefitinib [32]. Also, using NSCLC cell, INCB3619 increased apoptosis and reduced the apoptotic threshold for response to paclitaxel [32]. Consistent with this finding, the inhibitor decreased tumour growth and enhanced the therapeutic benefit of paclitaxel in a xenograft model of these cells.

As well as lung cancer, INCB3619 has also been shown to block the growth of breast cancer. Thus, INCN3619 was shown to synergise with paclitaxel in inhibiting growth of breast cancer in a xenograft model [32]. In a different study, although the addition of this compound to MCF-7 breast cancer cells in vitro resulted in minimal growth inhibition, when combined with the dual EGFR/HER2 tyrosine kinase inhibitor, GW2974 (Sigma Aldrich), synergistic growth inhibition was observed [33]. The combination of INCB3619 and GW2974 also gave rise to decreased phosphorylation of ERK and AKT,

Table 2 Selective ADAM inhibitors

\begin{tabular}{llll}
\hline Inhibitor & Target ADAM & Company & Refs \\
\hline INCB3619 & 10,17 & Incyte, Wilmington, DE & {$[32-34]$} \\
\hline INCB7839 & 10,17 & Incyte & {$[34,70-72]$} \\
\hline WAY-022 & 17 & Wyeth-Aherst, Pearl River, NY & {$[73]$} \\
\hline GI254023X & 10 & Glaxo Smith Kline & {$[74]$} \\
\hline GW280264X & 10,17 & Glaxo Smith Kline & {$[74]$} \\
\hline TMI-2 & 17 & Pfizer & {$[75]$} \\
\hline KB-R7785 & 12 & Organon, Osaka, Japan & {$[80]$} \\
\hline
\end{tabular}


suggesting blockage of the MAPK pathway. Using a xenograft breast cancer model, an inhibitor related to INCB3619, i.e., INCB7839 was found to decrease tumour volume [34]. However, when combined with the tyrosine kinase inhibitor, lapatinib, complete inhibition of tumour growth was observed. An important finding with the animal models investigated was that administration of INCB3619, in contrast to previous studies with MMP inhibitors [76,77], did not appear to induce musculoskeletal side effects [33].

INCB7839 is currently undergoing early clinical trials in HER2-positive advanced breast cancer patients [71,72]. Preliminary results suggest that this drug is generally well tolerated with no significant adverse effects that might be expected from inhibition of MMPs (musculoskeletal side effects) or EGFR-related kinases (skin rash). Furthermore, there was no evidence of drug-induced increases in liver enzymes, bone marrow toxicity or increase in cardiomyopathy. In a recently reported abstract [72], administration of INCB7839 and trastuzumab to 51 patients with advanced HER2-positive breast cancer induced response in 13/26 (50\%) evaluable patients. In 14 patients where the INCB7839 plasma concentration exceeded the $\mathrm{IC}_{50}$ for HER2 cleavage, response was obtained in 9 (64\%). Previous studies had shown that administration of trastuzumab monotherapy to patients with advanced breast cancer induced response rates of approximately $14-35 \%$ [78,79]. Thus, it would appear that the addition of INCB7839 to trastuzumab increased efficacy. Phase III clinical trials however, will be necessary to confirm this finding.

\section{Potential side effects from anti-ADAM treatments}

Most of our information on the potential side-effects of anti-ADAM inhibitors has been derived from clinical trials involving the use of ADAM17 inhibitors to treat patients with rheumatoid arthritis $[68,69]$. Unfortunately, most of these studies were limited to phase I and phase II trials because of lack of efficacy and/or hepatotoxicity. The origin of the hepatotoxicity is unclear. However, as mentioned above, early results from phase I/II trials with the dual ADAM10/17 inhibitor INCB7839 do not suggest major toxicity problems with this agent. As mentioned above, a particularly encouraging finding with INCB3619 and INCB7839 is that they do not appear to cause musculoskeletal side effects [33], which was a major problem with the early metalloproteinase inhibitors investigated [76,77]. Continued caution however, with respect to toxicity, will be necessary, especially as ADAM10 and ADAM17 act on wide variety of membrane proteins $[1,2]$.

\section{Acknowledgements}

The authors wish to thank Science Foundation Ireland, Strategic Research Cluster Award [08/SRC/B1410] to Molecular Therapeutics for Cancer Ireland for funding this work.

Author details

${ }^{1}$ Department of Pathology and Laboratory Medicine, St. Vincent's University Hospital, Dublin 4, Ireland. ${ }^{2}$ UCD School of Medicine and Medical Science, Conway Institute of Biomolecular and Biomedical Research, University College Dublin, Dublin 4, Ireland. ${ }^{3}$ National Institute for Cellular Biotechnology, Dublin City University, Dublin 9, Ireland. ${ }^{4}$ Department of Medical Oncology, St Vincent's University Hospital, Dublin 4, Ireland.

\section{Authors' contributions}

MJD researched the literature, conceived and wrote the manuscript. PMcG designed and formatted Figure 1. All authors read, edited and agreed with the content.

\section{Competing interests}

The authors declare that they have no competing interests. 


\section{References}

1. Edwards DR, Handsley MH, Pennington CJ: The ADAM metalloproteinases. Mol Aspects Med 2008, 29:258-89.

2. Murphy G: The ADAMs: Signaling scissors in the tumour microenvironment. Nat Rev Cancer 2008, 8:929-41.

3. Blobel CP: ADAMS: key components in EGFR signalling and development. Nature Rev Cancer 2005, 6:32-43.

4. Duffy MJ, McKiernan E, O'Donovan N, McGowan P: Role of ADAMs in cancer formation and progression. Clin Cancer Res 2007, 13:2335-2343

5. Duffy MJ, McKiernan E, O'Donovan N, McGowan P: The role of ADAMs in disease pathophysiology. Clin Chim Acta 2009, 403:31-36.

6. Gonzales PE, Solomon A, Miller AB, et al: Inhibition of tumor necrosis factor-a-converting enzyme by its prodomain. J Biol Chem 2004, 279:31638-31645.

7. Stupack DG: The Biology of integrins. Oncology [Williston Park] 2007, 21:6-12.

8. Arribas J, Bech-Serra JJ, Santiago-Josefat B: ADAMs, cell migration and cancer. Cancer Met Rev 2006, 25:57-68.

9. Reiss K, Ludwig A, Saftig P: Breaking up the tie: disintegrin-like metalloproteinases as regulators of cell migration in inflammation and invasion. Pharmacol Ther 2006, 111:985-1006.

10. Reddy P, Slack JL, Davis R: Functional analysis of the domain structure of tumor necrosis factor-alpha converting enzyme. J Biol Chem 2000, 275:14608-14.

11. Janes PW, Saha N, Barton WA, et al: Adam meets Eph: an ADAM substrate recognition module acts as a molecular switch for ephrin cleavage in trans. Cell 2005, 123:291-304.

12. Díaz-Rodríguez E, Montero JC, Esparís-Ogando A, Yuste L, Pandiella A: Extracellular signal-regulated kinase phosphorylates tumor necrosis factor alpha-converting enzyme at threonine 735: a potential role in regulated shedding. Mol Biol Cell 2002, 13:2031-44

13. Xu P, Derynck R: Direct activation of TACE-mediated ectodomain shedding by p38 MAP kinase regulates EGF receptor-dependent cell proliferation. Mol Cell 2010, 37:551-566.

14. Poghosyan Z, Robbins SM, Houslay MD, et al: Phosphorylation-dependent interactions between ADAM15 cytoplasmic domain and Src family protein-tyrosine kinases. J Biol Chem 2002, 277:4999-5007.

15. Kenny PA, Bissell MJ: Targeting TACE-dependent EGFR ligand shedding in breast cancer. J Clin Invest 2007 117:337-345

16. McGowan PM, Ryan BM, Hill AD, McDermott E, O'Higgins N, Duffy MJ: ADAM-17 expression in breast cancer correlates with variables of tumor progression. Clin Cancer Res 2007, 13:2335-2343.

17. Borrell-Pages M, Rojo F, Albanell J, Baselga J, Arribas J: TACE is required for the activation of the EGFR by TGF-alpha in tumors. EMBO J 2003, 22:1114-1124.

18. Peduto L, Reuter VE, Sehara-Fujisawa A, Shaffer DR, Scher HI, Blobel CP: ADAM12 is highly expressed in carcinomaassociated stroma and is required for mouse prostate tumor progression. Oncogene 2006, 25:5462-5466.

19. Franovic A, Robert I, Smith K, et al: Multiple acquired renal carcinoma tumor capabilities abolished upon silencing of ADAM17. Cancer Res 2006, 66:8083-8090.

20. Takamune $Y$, Ikebe T, Nagano O, Shinohara M: Involvement of NF-kappaB-mediated maturation of ADAM-17 in the invasion of oral squamous cell carcinoma. Biochem Biophys Res Commun 2008, 365:393-398.

21. Zheng $X$, Jiang $F$, Katakowski M, et al: ADAM17 promotes breast cancer cell malignant phenotype through EGFRPI3K-AKT activation. Cancer Biol Ther 2009, 8:1045-1054

22. Guaiquil V, Swendeman S, Yoshida T, Chavala S, Campochiaro PA, Blobel CP: ADAM9 is involved in pathological retinal neovascularization. Mol Cell Biol 2009, 29:2694-703.

23. Horiuchi K, Weskamp G, Lum L, et al: Potential role for ADAM15 in pathological neovascularization in mice. Mol Cell Biol 2003, 23:5614-24.

24. Mochizuki S, Tanaka R, Shimoda M, et al: Connective tissue growth factor is a substrate of ADAM28. Biochem Biophys Res Commun 2010, 402:651-7.

25. Weskamp G, Mendelson K, Swendeman S, Le Gall S, Ma Y, Lyman S, Hinoki A, Eguchi S, Guaiquil V, Horiuchi K, Blobel CP: Pathological neovascularization is reduced by inactivation of ADAM17 in endothelial cells but not in pericytes. Circ Res 2010, 106:932-40

26. Valkovskaya $\mathrm{N}$, Kayed $\mathrm{H}$, Felix $\mathrm{K}$, et al: ADAM8 expression is associated with increased invasiveness and reduced patient survival in pancreatic cancer. J Cell Mol Med 2007, 11:1162-1174.

27. Roemer A, Schwettmann L, Jung M, et al: Increased mRNA expression of ADAMs in renal cell carcinoma and their association with clinical outcome. Oncol Rep 2004, 11:529-536.

28. Fritzsche FR, Jung M, Tolle A, et al: ADAM9 Expression is a Significant and Independent Prognostic Marker of PSA Relapse in Prostate Cancer. Eur Urol 2007, 54:1097-106.

29. Zhong JL, Poghosyan Z, Pennington CJ, et al: Distinct Functions of Natural ADAM-15 Cytoplasmic Domain Variants in Human Mammary Carcinoma. Mol Cancer Res 2008, 6:383-394.

30. O'Shea C, McKie N, Buggy Y, Duggan C, Hill AD, McDermott E, O'Higgins N, Duffy MJ: Expression of ADAM9 mRNA and protein in breast cancer. Int J Cancer 2003, 105:754-61.

31. McGowan PM, McKiernan E, Bolster F, et al: ADAM-17 predicts adverse outcome in patients with breast cancer. Ann Oncol 2008, 19:1075-81

32. Zhou B-B S, Petyon M, He B, et al: Targeting ADAM-mediated ligand cleavage to inhibit HER3 and EGFR pathways in non-small cell lung cancer. Cancer Cell 2006, 10:39-50

33. Fridman JS, Caulder E, Hansbury M, et al: Selective inhibition of ADAM metalloproteases as a novel approach for modulating ErbB pathways in cancer. Clin Cancer Res 2007, 13:1892-902.

34. Witters $L$, Scherle P, Friedman S, et al: Synergistic Inhibition with a dual epidermal growth factor receptor/HER-2/neu tyrosine kinase inhibitor and a disintegrin and metalloprotease inhibitor. Cancer Res 2008, 68:7083-7089.

35. Sahin U, Weskamp G, Kelly K, et al: Distinct roles for ADAM10 and ADAM17 in ectodomain shedding of six EGFR ligands. J Cell Biol 2004, 164:769-779.

36. Sahin U, Blobel CP: Ectodomain shedding of the EGF-receptor ligand epigen is mediated by ADAM17. FEBS Lett 2007, 581:41-44.

37. Horiuchi K, Le Gall S, Schulte M, et al: Substrate selectivity of epidermal growth factor-receptor ligand sheddases and their regulation by phorbol esters and calcium influx. Mol Biol Cell 2007, 18:176-88. 
38. Peeters M, Price T, Van Laethem JL: Anti-epidermal growth factor receptor monotherapy in the treatment of metastatic colorectal cancer: where are we today? Oncologist 2009, 14:29-39.

39. Browne BC, O'Brien N, Duffy MJ, et al: HER-2 signaling and inhibition in breast cancer. Current Cancer Drug Ther 2009, 9:419-38.

40. Bublil EM, Yarden Y: The EGF receptor family: spearheading a merger of signaling and therapeutics. Curr Opin Cell Biol 2007, 19:124-34.

41. Singh B, Schneider M, Knyazev P, Ullrich A: UV-induced EGFR signal transactivation is dependent on proligand shedding by activated metalloproteases in skin cancer cell lines. Int J Cancer 2009, 124:531-539.

42. Mendelson K, Swendeman S, Saftig P, Blobel CP: Stimulation of platelet-derived growth factor receptor beta [PDGFRbeta] activates ADAM17 and promotes metalloproteinase-dependent cross-talk between the PDGFRbeta and epidermal growth factor receptor [EGFR] signaling pathways. J Biol Chem 2010, 285:25024-32.

43. Nanba D, Mammoto A, Hashimoto K, et al: Proteolytic release of the carboxy-terminal fragment of proHB-EGF causes nuclear export of PLZF. J Cell Biol 2003, 163:489-502.

44. Shimura T, Kataoka H, Ogasawara N, et al: Suppression of proHB-EGF carboxy-terminal fragment nuclear translocation: a new molecular target therapy for gastric cancer. Clin Cancer Res 2008, 14:3956-65.

45. Ikushima H, Miyazono K: TGFbeta signalling: a complex web in cancer progression. Nat Rev Cancer 2010, 10:415-24, Review.

46. Liu C, Xu P, Lamouille S, Xu J, Derynck R: TACE-mediated ectodomain shedding of the type I TGF-beta receptor downregulates TGF-beta signaling. Mol Cell 2009, 35:26-36.

47. Maretzky T, Reiss K, Ludwig A et al: ADAM10 mediates E-cadherin shedding and regulates epithelial cell-cell adhesion, migration, and $\beta$-catenin translocation. Proc Natl Sci USA 2005, 102:9182-9187.

48. Najy AJ, Day KC, Day ML: The ectodomain shedding of E-cadherin by ADAM15 supports ErbB receptor activation. J Biol Chem 2008, 283:18393-401.

49. Schors K, Evan G: Tumor angiogenesis: cause or consequence of cancer? Cancer Res 2007, 67:7059-61.

50. Zhao J, Chen H, Peschon JJ, et al: Pulmonary hypoplasia in mice lacking tumor necrosis factor-alpha converting enzyme indicates an indispensable role for cell surface protein shedding during embryonic lung branching morphogenesis. Dev Biol 2001, 232:204-218.

51. Gooz P, Gooz M, Baldys A, et al: ADAM-17 regulates endothelial cell morphology, proliferation and in vitro angiogenesis. Biochem Biophys Res Commun 2009, 380:33-8.

52. Duffy MJ: Clinical uses of tumor markers: a critical review. Crit Rev Clin Lab Sci 2001, 38:225-262.

53. Roy $R$, Wewer UM, Zurakowski D, Pories SE, Moses MA: ADAM 12 cleaves extracellular matrix proteins and correlates with cancer status and stage. J Biol Chem 2004, 279:51323-30.

54. Pories SE, Zurakowski D, Roy R, Lamb CC, Raza S, Exarhopoulos A, Scheib RG, Schumer S, Lenahan C, Borges V, Louis GW, Anand A, Isakovich N, Hirshfield-Bartek J, Wewer U, Lotz MM, Moses MA: Urinary metalloproteinases: noninvasive biomarkers for breast cancer risk assessment. Cancer Epidemiol Biomarkers Prev 2008, 17:1034-42.

55. Duffy MJ: Serum tumor markers in breast cancer: are they of clinical value? Clin Chem 2006, 52:345-51.

56. Fröhlich C, Albrechtsen R, Dyrskjøt L, Rudkjaer L, Ørntoft TF, Wewer UM: Molecular profiling of ADAM12 in human bladder cancer. Clin Cancer Res 2006, 12:7359-68.

57. Kuroda H, Mochizuki S, Shimoda M, Chijiiwa M, Kamiya K, Izumi Y, Watanabe M, Horinouchi H, Kawamura M, Kobayashi K, Okada Y: ADAM28 is a serological and histochemical marker for non-small-cell lung cancers. Int J Cancer 2010.

58. Grützmann R, Lüttges J, Sipos B, Ammerpohl O, Dobrowolski F, Alldinger I, Kersting S, Ockert D, Koch R, Kalthoff H, Schackert HK, Saeger HD, Klöppel G, Pilarsky C: ADAM9 expression in pancreatic cancer is associated with tumour type and is a prognostic factor in ductal adenocarcinoma. Br J Cancer 2004, 90:1053-8.

59. Duffy MJ: Predictive markers in breast and other cancers: a review. Clin Chem 2005, 51:494-503.

60. Sieuwerts AM, Meijer-van Gelder ME, Timmermans M, Trapman AM, Garcia RR, Arnold M, Goedheer AJ, Portengen H, Klijn JG, Foekens JA: How ADAM-9 and ADAM-11 differentially from estrogen receptor predict response to tamoxifen treatment in patients with recurrent breast cancer: a retrospective study. Clin Cancer Res 2005, 11:7311-21.

61. Moss ML, Bomar M, Liu Q, et al: The ADAM10 prodomain is a specific inhibitor of ADAM10 proteolytic activity and inhibits cellular shedding events. J Biol Chem 2007, 282:35712-21.

62. Gonzales PE, Solomon A, Miller AB, et al: J Biol Chem 2004, 279:31638-31645, Kveiborg M, Jacobsen J, Lee M-H, et al. Inhibition of the tumor necrosis factor-alpha-converting enzyme by its pro domain. J Biol Chem 2004, 279:31638-45.

63. Rapti M, Atkinson SJ, Lee MH, Trim A, Moss M, Murphy G: The isolated N-terminal domains of TIMP-1 and TIMP-3 are insufficient for ADAM10 inhibition. Biochem J 2008, 411:433-9.

64. Kveiborg M, Jacobsen J, Lee MH, Nagase H, Wewer UM, Murphy G: Selective inhibition of ADAM12 catalytic activity through engineering of tissue inhibitor of metalloproteinase 2 [TIMP-2]. Biochem J 2010, 430:79-86.

65. Lendeckel $U$, Kohl J, Arndt M, et al: Increased expression of ADAM family members in human breast cancer and breast cancer cell lines. J Clin Res Clin Oncol 2005, 131:41-8.

66. Gilmore JL, King BW, Harris K, et al: Synthesis and structure-activity relationship of a novel achiral series of TNFalpha converting enzyme inhibitors. Bioorganic Med Chem 2006, 16:2699-704.

67. Levin Jl, Chen JM, Laakso LM, et al: Acetylenic TACE inhibitors. Part 3: thiomorpholine sulfonamide hydroxamates. Bioorganic Med Chem Lett 2006, 16:1605-09.

68. Moss ML, Sklair-Tavron L, Nudelman R: Drug insight: tumor necrosis factor-converting enzyme as a pharmaceutical target for rheumatoid arthritis. Nat Clin Pract Rheumatol 2008, 4:300-9.

69. Moss ML, Stoeck A, Yan W, Dempsey PJ: ADAM10 as a target for anti-cancer therapy. Curr Pharm Biotechnol 2008, 9:2-8.

70. Fridman JS, Scherle PA, Liu X, et al: Preclinical characterization of INCB7839, a potent and selective inhibitor of ErbB ligand and HER2 receptor shedding: inhibition of ADAM10 and ADAM17 for the treatment of breast cancer. Breast Cancer Res Treat 2007, 106(Supp1):S82. 
71. Infante J, Burris HA, Lewis N, et al: A multicenter phase lb study of the safety, pharmacokinetics, biological activity and clinical efficacy of INCB7839, a potent and selective inhibitor of ADAM10 and ADAM17. Breast Cancer Res Treat 2007, 106(Supp1):S269.

72. Newton RC, Bradley EC, Levy RS, et al: Clinical benefit of INCB7839, a potential and selective ADAM inhibitor, in combination with trastuzumab in patients with metastatic HER2-positive breast cancer. J Clin Oncol 2010, 28(Suppl; abst 3025):7s.

73. Merchant NB, Voskresensky I, Rogers CM, et al: TACE/ADAM-17: a component of epidermal growth factor receptor axis and a promising therapeutic target in colorectal cancer. Clin Cancer Res 2008, 14:1182-191.

74. Hundhausen C, Misztela D, Berkhout TA, Broadway N, Saftig P, Reiss K, Hartmann D, Fahrenholz F, Postina R, Matthews V, Kallen KJ, Rose-John S, Ludwig A: The disintegrin-like metalloproteinase ADAM10 is involved in constitutive cleavage of CX3CL1 [fractalkine] and regulates CX3CL1-mediated cell-cell adhesion. Blood 2003, 102:1186-95.

75. Zhang $Y$, Hegen $M, X u$ J, et al: Characterization of [2R, 3S]-2-[[[4-[2-butynyloxy]phenyl]sulfonyl]amino]-N,3dihydroxybutanamide, a potent and selective inhibitor of TNF-alpha converting enzyme. Int Immunopharmacol 2004, 4:1845-1857.

76. Fingleton B: Matrix metalloproteinases as valid clinical targets. Current Pharm Design 2007, 13:333-46

77. Zucker S, Cao J, Chen W-T: Critical appraisal of the use of matrix metalloproteinases inhibitors in cancer treatment. Oncogene 2000, 19:6642-650.

78. Baselga J, Tripathy D, Mendelsohn J, et al: Phase II study of weakly intravenous recombinant humanized antip185HER2 monoclonal antibody in patients with HER2/neu overexpressing metastatic breast cancer. J Clin Oncol $1996,14: 737-744$

79. Cobleigh MA, Vogel CL, Tripathy D, Robert NJ, Scholl S, Fehrenbacher L, et al: Multinational study of the efficiacy and safety of humanised anti-HER2 monoclonal antibody in women who have HER2 overexpressing metastatic breast cancer that has progressed after chemotherapy for metastatic disease. J Clin Oncol 1999, 17:2639-2648.

80. Asakura M, Kitakaze M, Takashima S, et al: Cardiac hypertrophy is inhibited by antagonism of ADAM12 processing of HB-EGF: metalloproteinase inhibitors as a new therapy. Nat Med 2002, 8:35-40.

doi:10.1186/1559-0275-8-9

Cite this article as: Duffy et al:: The ADAMs family of proteases: new biomarkers and therapeutic targets for cancer? Clinical Proteomics 2011 8:9.

\section{Submit your next manuscript to BioMed Central} and take full advantage of:

- Convenient online submission

- Thorough peer review

- No space constraints or color figure charges

- Immediate publication on acceptance

- Inclusion in PubMed, CAS, Scopus and Google Scholar

- Research which is freely available for redistribution 